Canadian Oncology

Nursing Journal

Revue canadienne

de soins infirmiers

en oncologie

Volume 31, Issue 1 • Winter 2021

elSSN: 2368-8076 


\section{Acquisition de compétences en coordination des greffes de cellules souches hématopoiétiques : efficacité d'un parcours d'apprentissage pour les infirmières novices}

\section{par Cheryl Page, Charissa Cordon et Jiahui Wong}

RÉSUMÉ

L'augmentation du nombre de patients admissibles à recevoir une greffe de cellules souches hématopoḯtiques (GCSH) (ACO/CCO, 2017) entraîne un besoin accru d'infirmières spécialistes, la coordination des GCSH étant un rôle hautement spécialisé; il n'y a pourtant que peu de recherches portant sur la formation des infirmières. Habituellement, cette formation se fait par préceptorat, sans processus d'orientation formel.

Objectifs : Pour la présente étude pilote, un parcours d'apprentissage et des outils pédagogiques ont été élaborés en complément d'une approche systématique normalisée de la formation du personnel, et ce, dans le but d'améliorer la qualité des soins.

Méthodologie : Huit infirmières, sans expérience en coordination des GCSH, ont participé à l'étude et rempli les évaluations de suivi. Elles ont été évaluées avant l'intervention pour vérifier leurs

\section{AUTEURES}

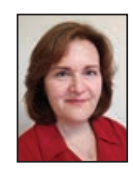

Cheryl Page, inf. aut., B.Sc.inf., B.Sc., M.Ed., CVAA(c), CSIO(C), BMTCN®, PIA (De Souza)

Clinicienne en éducation et perfectionnement pour les programmes d'hématologie et transplantation

Centre des sciences de la santé d'Hamilton

Courriel : pagec@hhsc.ca

Adresse : Unité C4, Centre hospitalier et de cancérologie Juravinski du Centre des sciences de la santé d'Hamilton 711 Concession St., Hamilton (Ontario) L8V 1 C3

Tél. : 905-521-2100, poste 42195

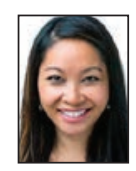

Charissa Cordon, inf. aut., M.Sc.inf., D.Éd.

Infirmière-chef

Centre des sciences de la santé d'Hamilton

Adresse : $1 \mathrm{~F}-24$

1200 Main St. W, Hamilton (Ontario) L8N $3 Z 5$

Centre médical de l'Université McMaster

Tél. : 905-521-2100, poste 73415

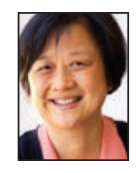

Jiahui Wong, Ph.D.

Gestionnaire, Évaluation des cours et programmes

Institut de Souza, Réseau universitaire de santé

Adresse : LuCliff Place, Suite 1903

700 Bay Street

Toronto (Ontario) M5G $1 Z 6$

Tél. : 416-581-7886

Centre des sciences de la santé d'Hamilton, Centre hospitalier et de cancérologie Juravinski

Programme de greffe de cellules souches hématopoïétiques

Hamilton (Ontario) connaissances ainsi que leurs lacunes pour chaque sous-rôle de la coordination de l'allogreffe et de la greffe autologue. Après cette évaluation, les infirmières recevaient une trousse d'étude autonome personnalisée respectant les étapes du parcours d'apprentissage avec un outil d'évaluation des compétences. Fondée sur les compétences, la formation se faisait "par modules », c'est-à-dire que les infirmières devaient maîtriser une compétence avant de passer à la suivante. Les infirmières étaient évaluées à l'aide du modèle de Kirkpatrick (2006) avant et après la période d'orientation pour voir l'évolution de leurs connaissances et de leurs comportements en coordination des greffes.

Résultats : Les participantes ont réagi favorablement aux outils et disent avoir grandement enrichi leurs connaissances et leurs compétences en coordination des greffes après la période d'orientation. Pour compléter la présente étude pilote, d'autres recherches devraient examiner l'utilisation d'un parcours d'apprentissage pour guider les nouvelles infirmières en GCSH dans leur rôle de coordination.

\section{INTRODUCTION}

$\mathrm{D}$ evant l'augmentation marquée du nombre de patients admissibles à recevoir une greffe de cellules souches hématopoiétiques (GCSH), davantage d'infirmières dûment formées en GCSH seront nécessaires. En Ontario, l'augmentation du nombre de patients admissibles résulte de multiples facteurs : avancées technologiques, augmentation des cas où la greffe est recommandée, élargissement des critères d'admissibilité et vieillissement de la population. En 2016 et 2017, 913 patients ont reçu une GCSH dans la province, et 45 autres patients ont subi une greffe à l'étranger (ACO/CCO, 2017), ce qui représente une augmentation de $79 \%$ depuis 2009-2010 (ACO/CCO, 2017). De plus, on s'attend à ce que cette augmentation s'accélère. Pour s'y préparer, de nombreux hôpitaux qui pratiquent des greffes de cellules souches hématopoiétiques, comme le Centre des sciences de la santé d'Hamilton (CSSH), améliorent leurs modèles de soins et intensifient la formation du personnel novice dans ce domaine. Actuellement, le CSSH est l'un des hôpitaux ontariens où l'on effectue les trois types de greffes (greffe autologue, allogreffe apparentée et allogreffe non apparentée). Tous les centres sont agréés par la Foundation for the Accreditation of Cellular Therapy (FACT).

L'allogreffe de cellules souches de sang périphérique fait appel à la chimiothérapie, avec ou sans radiothérapie, pour 
faire de la place aux cellules souches du donneur qui remplaceront le système lympho-hématopoiétique du patient afin d'aider à combattre la leucémie et de prévenir la récidive (Ezzone, 2013). En cas d'allogreffe de cellules souches hématopoïétiques, le donneur de cellules progénitrices et le receveur sont deux personnes différentes. Pour la greffe autologue de cellules souches de sang périphérique, on veut protéger les cellules souches hématopoíétiques du patient des effets de la chimiothérapie sur la moelle épinière. Ces cellules souches sont donc prélevées et mises en cryoconservation; le patient reçoit ainsi de fortes doses de chimiothérapie pour détruire la moelle épinière. Ensuite, on lui réimplante ses propres cellules souches pour rétablir la fonction hématopoiétique. Du point de vue hématologique, cette approche permet d'administrer des doses de chimiothérapie plus fortes que s'il n'y avait pas eu prélèvement de cellules souches (Ezzone, 2013). Dans une greffe autologue, le patient est à la fois donneur et receveur des cellules hématopoiétiques progénitrices. La coordination des greffes est un rôle infirmier hautement spécialisé qui requiert une bonne compréhension des troubles hématologiques, des traitements associés et de la trajectoire des soins pour ce groupe de patients. L'infirmière coordonnatrice organise le processus d'allogreffes en tenant compte des normes et du plan de soins du patient, notamment des résultats des examens médicaux administrés au donneur et au receveur dans les 30 jours précédant la date prévue de la greffe. L'infirmière coordonnatrice doit connaître les normes de qualité des soins de la FACT, ainsi que les points de repère provinciaux d'Action Cancer Ontario (ACO). La coordination des greffes doit être faite par des infirmières spécialisées, et ces dernières doivent être correctement formées à ce nouveau rôle afin de prodiguer efficacement ces soins particuliers. Toutefois, à l'heure actuelle, la recherche sur les pratiques d'orientation des infirmières qui coordonnent les GCSH comporte des lacunes.

La présente étude avait pour objectif d'explorer l'efficacité d'un parcours d'apprentissage pour compléter l'orientation, fondée sur les compétences, des infirmières du CSSH qui endossent pour la première fois le rôle de coordonnatrice des greffes. Ce programme d'orientation, qui suit les étapes du parcours d'apprentissage, met l'apprenant (et non l'instructeur) au centre de la démarche. Principalement, l'étude évaluait la perception, par les infirmières, de la trousse d'étude et de l'outil d'évaluation des compétences, tous deux élaborés conformément au parcours d'apprentissage et distribués aux nouvelles coordonnatrices de greffes du programme de thérapie cellulaire du CSSH pendant l'orientation. Voici les questions ayant guidé la recherche :

1. Dans quelle mesure la réaction des participantes au parcours d'apprentissage a-t-elle été favorable?

2. Dans quelle mesure les participantes ont-elles acquis les connaissances nécessaires à la coordination des greffes?

3. Dans quelle mesure les participantes ont-elles appliqué les apprentissages sur la coordination des greffes à leur nouveau rôle?
Habituellement, l'orientation à la coordination des greffes n'est pas guidée par un parcours d'apprentissage formel. Le CSSH a choisi d'élaborer des parcours d'apprentissage qui intègrent les normes de pratique de l'ACIO/CANO (2002) et de la FACT pour encadrer l'évaluation des besoins du personnel novice et s'assurer que toutes les notions sont bien maîtrisées. Ces parcours contribuent à la prestation des soins aux patients recevant une GCSH. Le nouveau personnel en coordination des allogreffes et des greffes autologues reçoit ainsi l'aide qui lui faut. Les parcours d'apprentissage s'accompagnent d'une trousse d'étude autonome et d'un outil d'évaluation des compétences pour chaque sous-rôle de la coordination des allogreffes et greffes autologues. Cette trousse d'étude guide l'infirmière coordonnatrice novice et sert de complément au plan d'apprentissage et à la vérification des acquis. Rempli par l'apprenante en consultation avec les responsables du préceptorat et de la formation de l'unité, l'outil d'évaluation des compétences permet de documenter et d'évaluer l'atteinte des compétences fondamentales nécessaires pour coordonner les GCSH.

\section{REVUE DE LA LITTÉRATURE}

$\mathrm{Vu}$ le peu de recherches menées sur la formation des infirmières au rôle de coordination des greffes, ce sont les méthodes employées dans le domaine médical qui ont été examinées. Schumacher et Risco (2017) définissent la compétence comme " un énoncé précisément formulé établissant un ou des résultats mesurables ou observables ». Depuis plus de vingt ans, l'apprentissage fondé sur les compétences est la norme dans le monde médical (Boyd et al, 2018). Greet et collaborateurs (2014) ont constaté que les résultats obtenus sont de loin supérieurs lorsque le nouveau personnel connaît les critères sur lesquels il est évalué (comme c'est le cas dans l'évaluation fondée sur les compétences) et que ces critères portent à la fois sur les connaissances théoriques et pratiques. L'outil d'évaluation des compétences du personnel de coordination des greffes expliquait le rôle et les ressources auxquelles le nouvel apprenant devait se référer pour acquérir les connaissances théoriques associées à une compétence donnée. Selon Schumacher et Risco (2017), l'orientation axée sur les compétences plutôt que sur le contenu constitue une approche fondée sur les résultats; l'apprentissage se fait «par modules », c'est-à-dire que la compétence doit être maîtrisée avant qu'une autre ne soit introduite, permettant au responsable du préceptorat de savoir ce qui pose problème à l'apprenant. Comme la coordination des greffes exige tout de même une connaissance de base des pathologies en cause, la trousse d'étude a été conçue pour aider les infirmières novices à comprendre leurs besoins en matière d'apprentissage et leur fournir des ressources complémentaires à la formation sur le déroulement et les processus de la coordination des greffes en tant que telle.

Selon Milgore et collaborateurs (2017), les parcours d'apprentissage exhaustifs améliorent la satisfaction et le moral du personnel, en plus d'en favoriser la rétention. Pour Johnston et Ferraro (2001), une orientation clinique systématique et complète est essentielle, et le parcours d'apprentissage sert de cadre à cette orientation. Le cadre d'orientation permet à 
l'apprenant et au responsable du préceptorat de planifier les objectifs d'acquisition des compétences selon un calendrier précis à partir de certains critères. Si les critères ne sont pas respectés dans les délais prescrits, cela peut signaler la présence de difficultés pour l'apprenant (Johnston et Ferraro, 2001). Le responsable de la formation de l'unité peut alors travailler avec le nouvel apprenant et le responsable du préceptorat pour établir un plan qui comblera les lacunes. Pour Bumgarner et Biggerstaff (2000), le parcours d'apprentissage sert de carte pour guider à la fois l'apprentissage et le préceptorat, en plus d'encourager la pensée critique, d'atténuer le choc de la réalité du nouveau rôle, et d'accroître la satisfaction au travail ainsi que la rétention du personnel. L'efficacité des parcours d'apprentissage est déjà bien établie. Grâce à la combinaison du parcours d'apprentissage et de l'apprentissage fondé sur les compétences, les nouvelles coordonnatrices des greffes sont mieux préparées à leur rôle et peuvent progresser efficacement pour dépasser le stade de novices.

\section{ÉLABORATION DU PARCOURS D'APPRENTISSAGE}

Par le passé, au CSSH, l'orientation à la coordination des greffes se faisait uniquement par préceptorat sans définition formelle des compétences, du calendrier ou du processus à respecter pour maîtriser les différentes étapes de l'apprentissage du nouveau rôle. Pour structurer la démarche d'orientation, le CSSH a donc élaboré, à partir des commentaires d'infirmières coordonnatrices d'expérience, le parcours d'apprentissage ainsi que la trousse d'étude et l'outil d'évaluation des compétences qui l'accompagnent. La procédure complexe de coordination des greffes a été divisée en plusieurs étapes.
Lorsque l'infirmière novice complète une étape et se sent à l'aise avec les tâches enseignées, elle passe à la suivante. À chaque étape, elle a le temps d'observer le travail du responsable du préceptorat, puis d'accomplir les mêmes tâches avec un degré d'aide variable. Elle gagne progressivement en indépendance jusqu'à pouvoir réaliser une tâche donnée avec peu ou pas d'assistance. Bien qu'il existe un calendrier pour guider la progression, le temps nécessaire pour passer d'une étape à l'autre varie selon les besoins de l'apprenant, qui sont évalués en collaboration avec le responsable du préceptorat. En consultation avec ce dernier, l'apprenant remplit aussi régulièrement l'outil d'évaluation des compétences pour s'assurer d'atteindre les objectifs de chaque étape du parcours. Dans l'outil, chaque étape s'accompagne de ressources suggérées et de résultats mesurables. La coordination de l'allogreffe (figure 1) et de la greffe autologue (figure 2) suivent deux parcours d'apprentissage différents.

Le modèle infirmier de Benner (1984) et les normes de l'Association canadienne des infirmières en oncologie (2002) servent de cadre pour classer les compétences selon différents niveaux (novice, débutant avancé, compétent, expérimenté et expert). Le modèle de Benner tient compte à la fois du rôle des connaissances et de celui des compétences pratiques acquises par l'expérience dans la formation des infirmières. Lorsqu'une infirmière experte en hématologie assume un rôle nouveau pour elle, comme celui de coordonnatrices des greffes, elle revient à un niveau de compétence moins avancé du modèle de Benner. Si certaines de ses compétences sont transférables, elle apprendra son nouveau rôle plus rapidement. Le parcours d'apprentissage est conçu pour aider l'infirmière en coordination des greffes à franchir les différents stades d'acquisition des compétences.

Figure 1. Parcours d'apprentissage en coordination des allogreffes

Parcours d'apprentissage en coordination des allogreffes

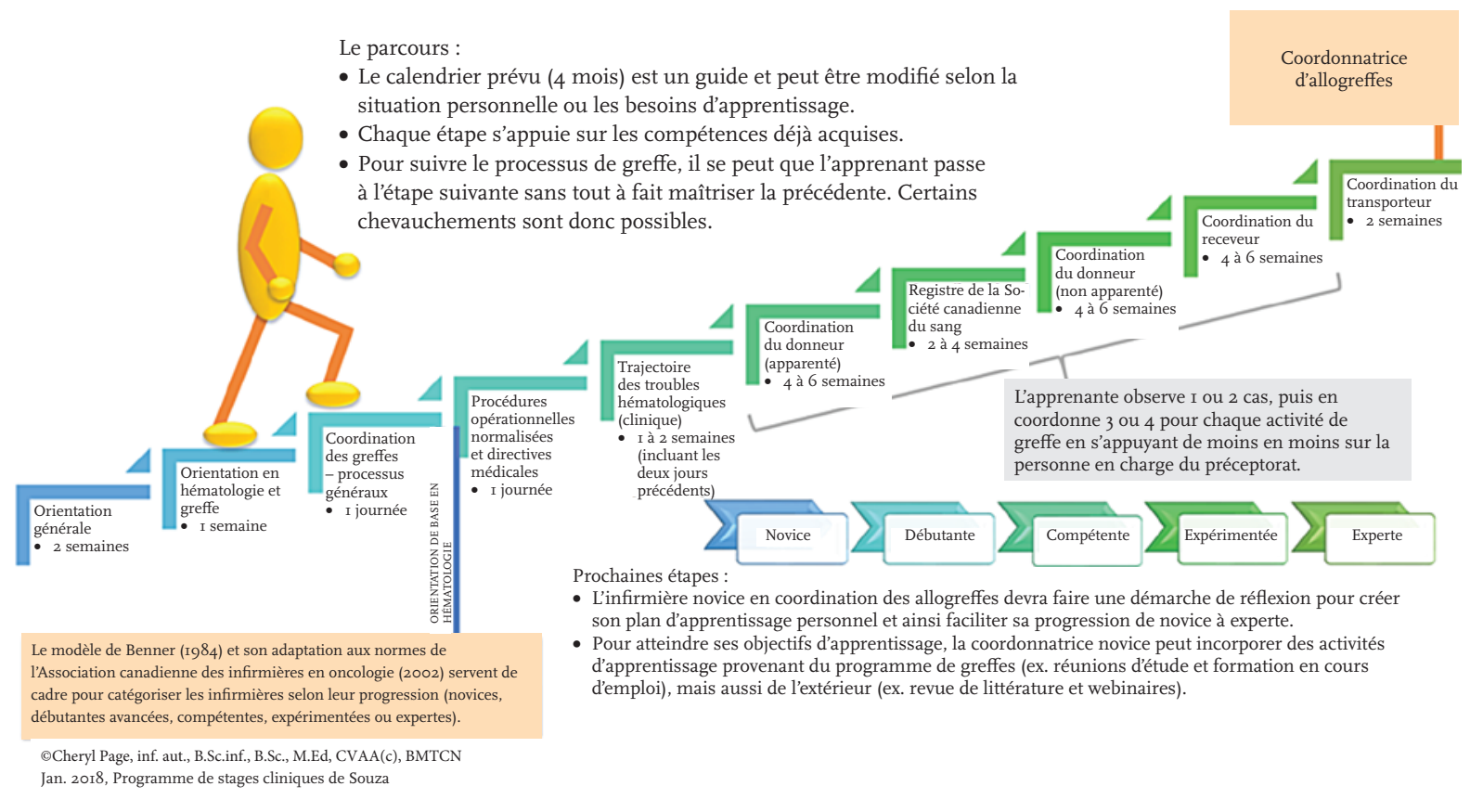


Figure 2. Parcours d'apprentissage en coordination des greffes autologues

Parcours d'apprentissage en coordination des allogreffes

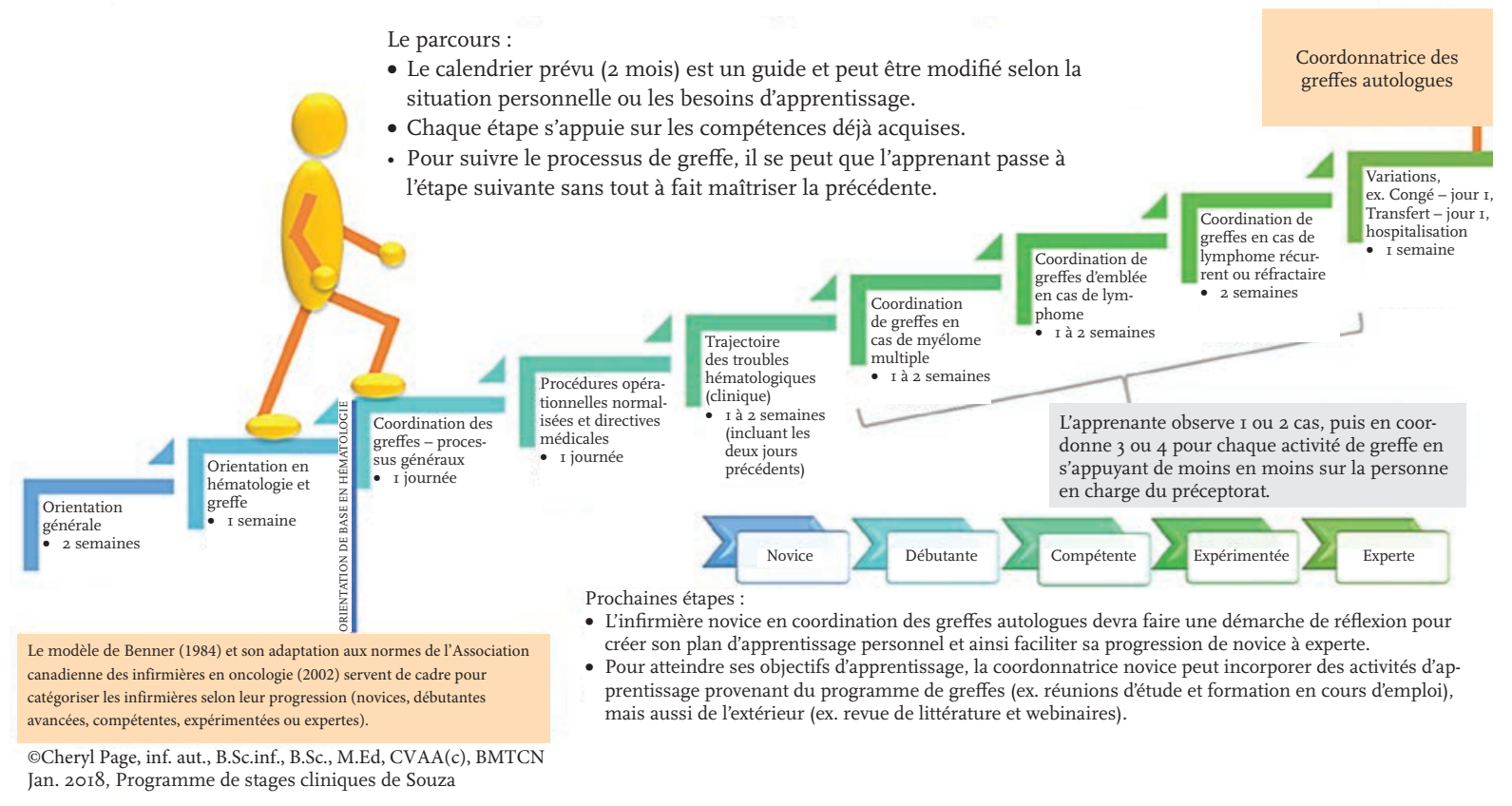

Le modèle de Benner comprend les niveaux suivants : novice, débutant avancé, compétent, expérimenté et, enfin, expert. Ce modèle a été adapté à la coordination des greffes. L'infirmière novice possède une compréhension conceptuelle de son rôle, une expérience clinique minimale et une connaissance limitée de la coordination des greffes, et sollicite des conseils pour la prise de décisions cliniques. Au niveau de débutante avancée, elle a une compréhension conceptuelle de son rôle, une expérience clinique minimale et une certaine connaissance de la coordination des greffes; son exposition aux situations cliniques est limitée, mais elle sait reconnaître les résultats normaux (Benner, 1984). L'infirmière compétente a une compréhension conceptuelle de son rôle et a eu l'occasion d'appliquer ses compétences en contexte clinique; exposée à de nombreuses situations, elle reconnaît les résultats normaux et anormaux, et sait établir les priorités et gérer des situations complexes (Benner, 1984). L'infirmière expérimentée a une compréhension conceptuelle de son rôle, ainsi que des compétences affirmées. Elle possède également de l'expérience clinique et son jugement clinique est rapide et précis. De plus, elle a été considérablement exposée à la plupart des situations; elle sait établir des priorités et peut prévoir l'évolution de l'état du patient (Benner, 1984). L'infirmière experte, quant à elle, synthétise l'information et la met en pratique. Elle dispose d'une grande compétence clinique, ses connaissances sont étendues et bien ancrées, et elle a été largement exposée à différents cas. Elle possède donc une compréhension profonde de la situation, peut cerner rapidement et systématiquement l'évolution actuelle de l'état du patient et établir les priorités rapidement, quelles que soient les conditions (Benner, 1984).

\section{MÉTHODOLOGIE}

La présente étude, qui fait appel à un échantillon de commodité, emploie un devis quasi-expérimental avec groupe de contrôle non équivalent de même qu'une approche par séries chronologiques.

\section{Participants}

L'échantillon était constitué d'infirmières en formation pour le rôle de coordonnatrice des greffes au CSSH. Pendant l'étude, huit infirmières ont complété le processus d'orientation, soit pour la coordination des allogreffes, soit pour la coordination des greffes autologues, et elles ont toutes accepté de participer à l'étude. Si une infirmière avait déjà été formée à la coordination des allogreffes ou greffes autologues par le passé et qu'elle refaisait le processus d'orientation pour l'autre type de greffes, elle était tout de même admissible à l'étude. Léchantillon regroupait donc $100 \%$ de la population cible. Pour faciliter le recrutement, une séance d'information était prévue pour expliquer l'intervention. Ensuite, les personnes intéressées fournissaient leur consentement éclairé à un membre du personnel qualifié qui ne faisait pas partie de l'équipe de coordination.

L'expérience des participantes en hématologie variait considérablement, allant de 4 à 30 ans d'expérience (14 en moyenne). Les participantes avaient travaillé dans des milieux variés, notamment les hôpitaux, les soins ambulatoires, l'unité d'aphérèse et les cliniques externes. Cinq étaient en orientation pour les greffes autologues et trois pour la coordination des allogreffes. Deux d'entre elles avaient déjà été formées en coordination des greffes autologues et voulaient maintenant devenir coordonnatrices des allogreffes. Aucune des participantes n'avait déjà suivi de formation en coordination des allogreffes. 


\section{Intervention}

Le parcours d'apprentissage, l'outil d'évaluation des compétences et la trousse d'étude ont formé la base du processus d'orientation. Les infirmières ont été informées qu'elles auraient accès à ces outils qu'elles décident ou non de prendre part à l'étude. Les infirmières ayant participé à l'étude ont rempli une évaluation avant la période d'orientation, puis de nouveau après. Le parcours d'apprentissage leur a été présenté comme un guide pour le processus d'orientation. La trousse d'étude fournie visait à faciliter le transfert des connaissances propres au nouveau rôle de coordination. L'outil d'évaluation des compétences, enfin, a servi à suivre les progrès des apprenantes.

\section{Mesures}

Le modèle de Kirkpatrick (2006) utilisé pour évaluer l'efficacité de la formation se décline en quatre niveaux. Le premier évalue si les participantes réagissent favorablement à la formation. Le deuxième examine le degré de maîtrise des connaissances, compétences ou attitudes enseignées pendant la formation. Le troisième vérifie le degré d'application dans le travail des apprentissages réalisés pendant la formation. Le quatrième évalue la mesure dans laquelle la formation a permis d'atteindre les objectifs ou résultats ciblés. Ce dernier niveau n'a pas été formellement évalué dans la présente étude.

Les questionnaires mesurant l'efficacité du programme de formation ont été adaptés afin d'évaluer les niveaux un, deux et trois du modèle de Kirkpatrick (2006). La mesure des objectifs et résultats ciblés de niveau quatre pour le rôle de coordonnatrice des greffes dépassait toutefois la portée de la présente étude.

\section{1) Niveau 1 : Dans quelle mesure la réaction des participantes au parcours d'apprentissage a-t-elle été favorable?}

La simplicité d'utilisation du parcours d'apprentissage, la valeur de l'information présentée dans la trousse d'étude, et l'exhaustivité de l'outil d'évaluation des compétences étaient mesurées à l'aide d'un questionnaire de satisfaction utilisant une échelle de Likert en 5 points (où $0=$ totalement en désaccord, et $5=$ totalement en accord). Le questionnaire était administré dès la fin du programme d'orientation.

\section{2) Niveau 2 : Dans quelle mesure les participantes ont-elles acquis les connaissances nécessaires à la coordination des greffes?}

Létendue des connaissances sur la coordination des greffes a été comparée à des données de base recueillies avant et après l'intervention d'orientation. Le questionnaire d'auto-évaluation des connaissances couvrait quatre aspects du processus de greffes : la procédure de GCSH, la place de la GCSH dans la trajectoire de soins du patient, les étapes des soins au patient pendant une GCSH, et l'organigramme de la coordination des GCSH. De plus, les apprenantes devaient attribuer une note au parcours d'apprentissage, à la trousse d'étude et à l'outil d'évaluation des compétences à l'aide d'une échelle de Likert en 5 points (où $0=$ faible valeur, et $5=$ valeur très élevée). Les participantes répondaient au questionnaire avant la période d'orientation, puis tout de suite après.
3) Niveau 3 : Dans quelle mesure les participantes ont-elles appliqué les apprentissages sur la coordination des greffes à leur nouveau rôle?

Lévolution des comportements en lien avec la coordination des greffes a été évaluée avant et après l'intervention. Le questionnaire d'auto-évaluation des comportements couvrait trois aspects importants des soins de coordination : la capacité de donner aux patients de l'enseignement sur le processus de GCSH, la capacité de coordonner la GCSH et le niveau de compétence selon le modèle de Benner (1984), mesuré sur une échelle de Likert en 5 points (où $0=$ très faible capacité et 5 = capacité très élevée).

\section{ANALYSE STATISTIQUE}

Les réponses des participantes étaient notées sur une échelle de Likert en 5 points. L'utilisation de données paramétrées à l'aide d'une échelle de Likert ou d'une méthode d'analyse non paramétrique ne fait pas l'unanimité (Sullivan et Artino, 2013). Selon Halter (2017) et Harpe (2015), les données évaluées selon une échelle de Likert en 5 points constituent en fait une mesure pondérée de la différence entre les choix de réponse, et peuvent donc être considérées comme des données continues. De leur côté, Sullivan et Artino (2013) recommandent, pour les données de type Likert, de comparer les moyennes, comme dans un test t, plutôt que les modes, comme dans un test des rangs signés, étant donné que les tests paramétriques sont plus puissants pour ce type de données que les tests non paramétriques lorsque l'échantillon comprend au moins 5 à 10 observations. Meek et collaborateurs (2007) ont soigneusement comparé le test t paramétrique au test des rangs signés de Wilcoxon pour le traitement des données de type Likert et ont constaté que le test t a une plus grande puissance, même pour de petits échantillons.

La différence entre les scores moyens obtenus avant et après l'intervention éducative a été analysée à l'aide d'un test t paramétrique bilatéral apparié. Dans une étude pilote exploratoire comme la nôtre, le test bilatéral permet de déterminer l'efficacité du parcours d'apprentissage pour guider l'orientation des infirmières novices en coordination des greffes. Les éléments de contenu de chaque niveau du modèle de Kirkpatrick ont été évalués séparément. Au premier niveau, la réaction au programme de formation n'a été mesurée qu'après l'intervention. Pour les niveaux deux (connaissances) et trois (comportements), les participantes devaient répondre aux mêmes questions avant et après le parcours d'apprentissage. On a ensuite comparé la moyenne des scores obtenus pour voir s'il y avait eu des changements.

La coordination des greffes est une sous-spécialité des soins infirmiers en hématologie, un domaine déjà hautement spécialisé. Majhail (2015) a examiné les modèles de soins de 85 centres de greffe de cellules souches aux ÉtatsUnis et constaté qu'il y avait en moyenne quatre coordonnateurs de greffes par établissement. Selon Hertzog (2008), une étude pilote doit rejoindre au moins $10 \%$ de la population concernée. Étant donné le peu de coordonnateurs dans les centres de GCSH, la taille de l'échantillon doit être supérieure à $10 \%$ pour que les résultats soient significatifs sur le plan 
statistique. Pour atténuer les erreurs d'échantillonnage et augmenter la précision lorsqu'on étudie de petites populations, Israel (2003) préconise de réaliser une enquête en utilisant la population entière comme échantillon. Dans la présente étude pilote, nous avions enrôlé $100 \%$ du nouveau personnel en formation pour le rôle de coordination. Par conséquent, les résultats montrent bien toute l'incidence de notre intervention au CSSH; ces résultats guideront les études menées dans d'autres centres.

\section{RÉSULTATS}

Huit infirmières ont accepté de participer à l'étude et d'en effectuer toutes les étapes. Aucune corrélation significative n'a été décelée entre les compétences que possédaient les infirmières en coordination des greffes avant $(r=.61, p=.112)$ ou après l'évaluation $(r=.42, p=.304)$ et les années d'expérience en hématologie. Il n'y avait pas non plus de corrélation significative entre les compétences des infirmières en coordination des greffes avant $(r=.19, p=.677)$ ou après l'évaluation $(r=.30$, $p=.513$ ) et l'expérience acquise précédemment dans un autre domaine de coordination.

\section{Premier niveau d'évaluation : la réaction}

Le premier niveau d'évaluation mesurait le degré de réaction favorable des participantes au parcours d'apprentissage et au matériel éducatif connexe (l'intervention). Les 8 participantes ont rempli le questionnaire postintervention. Les résultats montrent que $100 \%$ des participantes ont réagi favorablement au parcours d'apprentissage, c'est-à-dire que $37,5 \%$ (ou 3/8) d'entre elles étaient totalement d'accord pour dire que le parcours était facile à suivre et que $62,5 \%(5 / 8)$ étaient aussi d'accord avec cette affirmation. Le questionnaire a aussi montré que $75 \%$ (ou 6/8) des participantes étaient totalement d'accord et $25 \%$ (ou 2/8) en accord avec le fait que la trousse d'étude fournissait de l'information et des ressources précieuses. De plus, 62,5\% (ou 5/8) des participantes étaient totalement d'accord et 37,5\% (3/8) en accord pour dire que l'outil d'évaluation des compétences intégrait tous les aspects de la coordination.

\section{Deuxième niveau d'évaluation : les connaissances}

Un test $t$ d'échantillons appariés a permis de mesurer l'effet de l'intervention éducative sur les scores du personnel dans quatre domaines de connaissances. Pour l'auto-évaluation des connaissances sur la procédure de greffe, on a constaté une augmentation statistiquement significative des scores entre la pré-évaluation ( $\mathrm{M}=3,5$; ÉT $=0,53$ ) et la postévaluation $(\mathrm{M}=4,5$; ÉT $=0,53)$, pour $\mathrm{t}(7)=3,74$ et $p=.007$. La valeur de l'êta-carré $(0,7)$ montre une importante association.

Pour l'auto-évaluation des connaissances sur le rôle de la greffe dans la trajectoire de soins du patient, l'amélioration était également significative entre la pré-évaluation $(M=3,25$; ÉT $=0,46)$ et la postévaluation $(\mathrm{M}=4,63$; ÉT $=0,52)$, pour des valeurs de $\mathrm{t}(7)=7,51, p<.001$ et êta-carré de 0,9 .

Dans l'auto-évaluation des connaissances sur les étapes des soins au patient pendant le processus de greffe, on voit une augmentation statistiquement significative des scores entre la pré-évaluation $(\mathrm{M}=2,88$; ÉT $=0,64)$ et la postévaluation $(\mathrm{M}=4,50$; ÉT $=0,53)$, pour des valeurs de $\mathrm{t}(7)=6,18, p<.001$ et êta carré de 0,8 .

Pour l'auto-évaluation des connaissances sur l'organigramme de coordination pendant le processus de greffe, l'augmentation des scores était significative entre la pré-évaluation $(\mathrm{M}=1,75$, ÉT $=0,71)$ et la postévaluation $(\mathrm{M}=4,38$, ÉT $=0,74)$, pour des valeurs de $\mathrm{t}(7)=9,98, p<.001$ et êta-carré de 0,9 .

Pour vérifier les connaissances des apprenantes, on leur a demandé, avant et après l'intervention, d'auto-évaluer l'utilité du parcours d'apprentissage et des ressources connexes, ainsi que de la trousse d'étude et de l'outil d'évaluation des compétences pour guider leur orientation. La valeur accordée au parcours d'apprentissage n'avait pas changé entre la pré-évaluation $(M=4,63$; ÉT $=0,52)$ et la postévaluation $(M=4,63$; $\mathrm{ÉT}=0,52), \mathrm{t}(7)=0, p=\mathrm{ns}$. En ce qui concerne l'auto-évaluation de la trousse d'étude, aucun changement n'est survenu non plus entre la pré-évaluation ( $\mathrm{M}=4,75$; $\mathrm{E} T=0,46)$ et la postévaluation $(\mathrm{M}=4,88$; ÉT $=0,35)$, pour des valeurs de $\mathrm{t}(7)=0,55$ et $p=$ ns. Pour l'auto-évaluation de la valeur de l'outil d'évaluation des compétences, il n'y avait aucune augmentation statistiquement significative des scores entre la pré-évaluation $(M=4,75$; ÉT $=0,46)$ et la postévaluation $(\mathrm{M}=4,50$; ÉT $=0,53)$, pour des valeurs $\operatorname{det}(7)=0,80, p=\mathrm{ns}$.

En pré-évaluation, les participantes ont donné une note élevée à la valeur potentielle du parcours d'apprentissage : 37,5 \% étaient d'accord et $62,5 \%$ totalement d'accord pour dire que le parcours allait guider leur orientation. Les résultats de la postévaluation étaient les mêmes, les participantes ayant accordé une note élevée à la valeur réelle du parcours. De même, pour les documents connexes, $25 \%$ étaient d'accord et $75 \%$ totalement d'accord pour dire que la trousse d'étude guiderait leur orientation. En postévaluation, cette proportion passait à $12,5 \%$ de participantes en accord et de 87,5 \% totalement d'accord. Toutefois, cette augmentation n'était pas statistiquement significative. Pour l'outil d'évaluation des compétences, $25 \%$ des participantes étaient d'accord et $75 \%$ totalement d'accord pour dire qu'il allait guider leur orientation. Les proportions changeaient en postévaluation, passant à $50 \%$ en accord et $50 \%$ totalement d'accord, mais le changement n'était pas statistiquement significatif. Les participantes s'attendaient à ce que le parcours d'apprentissage et les ressources connexes guident leur orientation et elles n'ont pas changé d'avis après l'orientation.

\section{Troisième niveau d'évaluation : les comportements}

Un test $t$ d'échantillons appariés a permis de mesurer l'effet de l'intervention éducative sur les scores du personnel pour trois comportements. Dans l'auto-évaluation de la capacité à donner au patient de l'enseignement sur le processus de greffe, on a constaté une augmentation statistiquement significative des scores entre la pré-évaluation $(\mathrm{M}=2,63$; ÉT $=1,06)$ et la postévaluation ( $\mathrm{M}=4,88$; ÉT $=0,35)$, pour des valeurs de $\mathrm{t}(7)=6,15$ et $p<.001$. La valeur de l'êta-carré $(0,8)$ montre une importante association.

Dans l'auto-évaluation de la capacité à coordonner les différentes étapes du processus de greffe, l'augmentation des 
scores est statistiquement significative entre la pré-évaluation $(\mathrm{M}=1,75$; ÉT $=0,71)$ et la postévaluation $(\mathrm{M}=4,75$; ÉT $=0,46)$, pour des valeurs de $\mathrm{t}(7)=11,22$ et $p<.001$. La valeur de l'êta-carré $(0,9)$ montre une importante association.

Dans l'auto-évaluation des compétences infirmières, hiérarchisées selon le modèle de Benner, on a observé une augmentation statistiquement significative des scores entre la pré-évaluation $(\mathrm{M}=1,75$; ÉT $=0,71)$ et la postévaluation $(\mathrm{M}=3,50$; ÉT $=0,93)$, pour des valeurs de $\mathrm{t}(7)=7$ et $p<.001$. La valeur de l'êta-carré $(0,9)$ montre une importante association.

\section{DISCUSSION}

La présente étude pilote du programme de formation en coordination des GCSH montre que les participantes ont perçu favorablement le parcours d'apprentissage, ainsi que la trousse d'étude et l'outil d'évaluation des compétences (figure 3). Lorsqu'on évalue l'influence des facteurs démographiques sur les résultats, on constate que les années d'expérience acquise en hématologie ou dans un autre rôle de coordination n'ont pas d'effet significatif sur le niveau de compétences avant ou après l'intervention, ce qui s'explique probablement par la nature unique de ces rôles. Comme toutes les participantes possédaient une certaine expérience en hématologie, la différence était peu marquée. Si les participantes n'avaient pas eu la moindre expérience dans ce domaine, peutêtre les résultats auraient-ils été différents.

Les résultats de l'étude montrent une évolution importante des connaissances dans les domaines clés de la coordination des GCSH, notamment : la procédure de GCSH, la place de la GCSH dans la trajectoire de soins du patient, les étapes des soins au patient pendant une GCSH, et la coordination du donneur et du receveur pendant une GCSH (figure 4). Interrogées sur l'effet des ressources du programme

Figure 3. Premier niveau d'évaluation de Kirkpatrick : Réaction

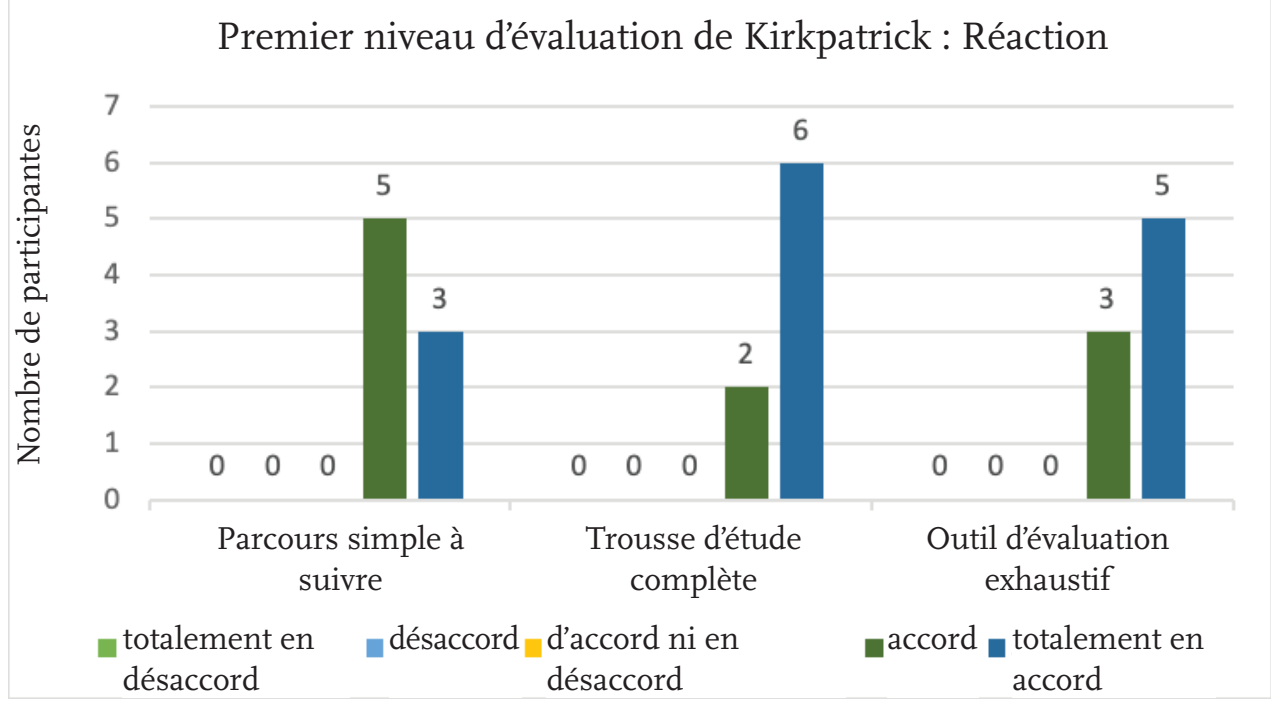

Figure 4. Évolution des connaissances avant et après le programme d'orientation

Deuxième niveau de Kirkpatrick : Connaissances avant et après l'intervention

Processus de greffe
Trajectoire du patient
Cheminement du
patient (étapes de soins)
Organigramme de
coordination

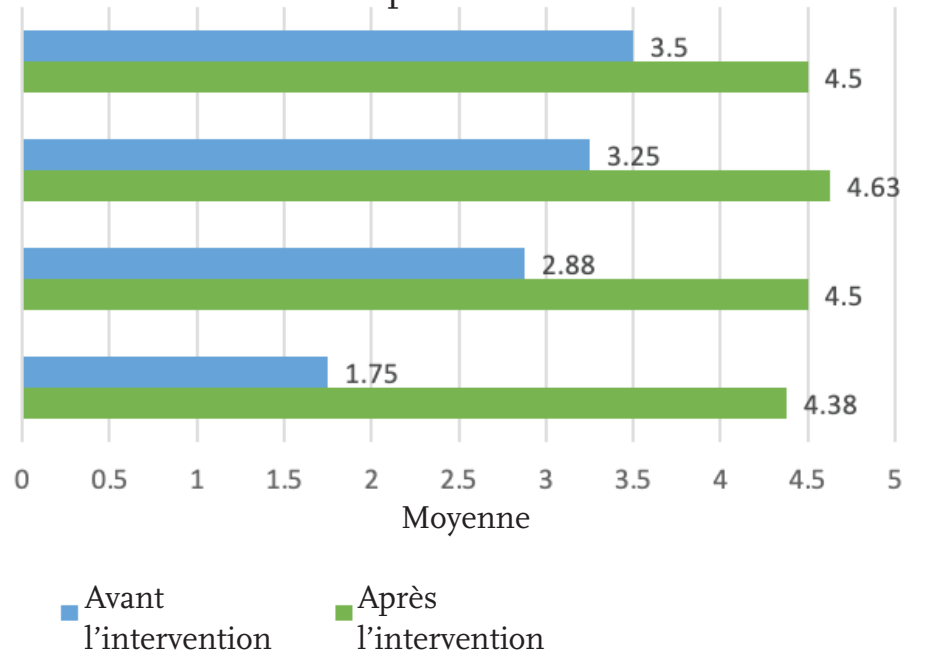


d'orientation sur l'acquisition des connaissances, les apprenantes ont accordé une note élevée à la valeur attendue du parcours d'apprentissage avant l'orientation, ainsi qu'à sa valeur réelle après l'orientation (figure 5). Les apprenantes ont donné une note élevée à la valeur de la trousse d'étude avant l'orientation, qu'elles ont majorée après l'orientation (figure 5). Elles ont aussi accordé une note élevée à la valeur de l'outil d'évaluation des compétences, avant et après l'orientation (figure 5). La valeur attribuée à ces ressources avant et après l'orientation ne présentait aucune différence statistiquement significative. Vraisemblablement, les attentes par rapport au parcours d'apprentissage, à la trousse d'étude et à l'outil d'évaluation des compétences étaient déjà élevées à la base, laissant peu de place à l'amélioration (un phénomène appelé « effet de plafonnement »). Ce point mériterait d'être exploré plus en profondeur.
Il s'est produit un changement significatif des comportements avant et après l'orientation, manifeste dans trois domaines clés de la coordination des greffes : la capacité d'offrir au patient de l'enseignement sur le processus de GCSH, la capacité de coordonner la GCSH, et le niveau de compétences selon le modèle de Benner (1984) (figure 6). Avant l'orientation, les apprenantes se qualifiaient surtout de novices ou de débutantes avancées (figure 6). Après l'orientation, la plupart se sentaient compétentes (figure 7). Au fur et à mesure qu'elles gagneront de l'expérience, les nouvelles coordonnatrices de GCSH continueront de franchir les différentes étapes du modèle de Benner (1984).

\section{Limites}

La présente étude comporte quelques limites. Le quatrième niveau d'évaluation du modèle de Kirkpatrick (2006), qui examine l'influence de l'intervention sur les résultats ciblés, n’a

Figure 5. Évaluation de la valeur du parcours d'apprentissage, de la trousse d'étude et de l'outil d'évaluation des compétences avant et après l'orientation

\section{Valeur accordée au parcours d'apprentissage et aux outils}

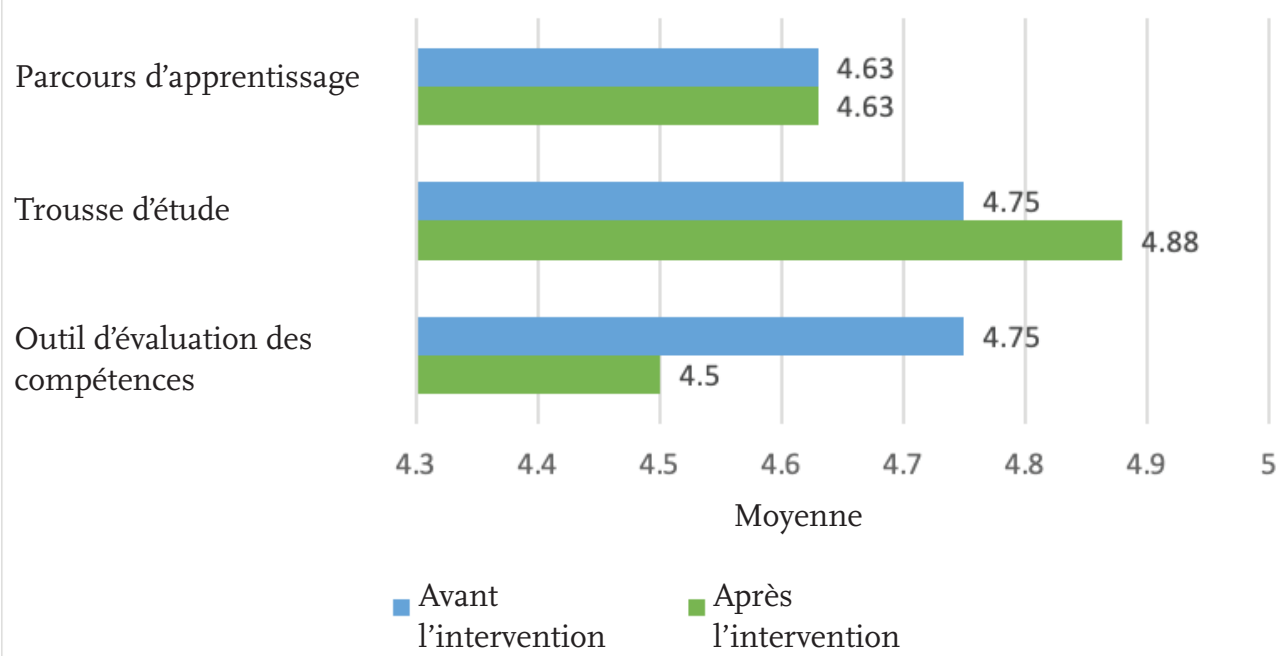

Figure 6. Évolution des comportements avant et après le programme d'orientation

Troisième niveau de Kirkpatrick : Comportements avant et après l'intervention

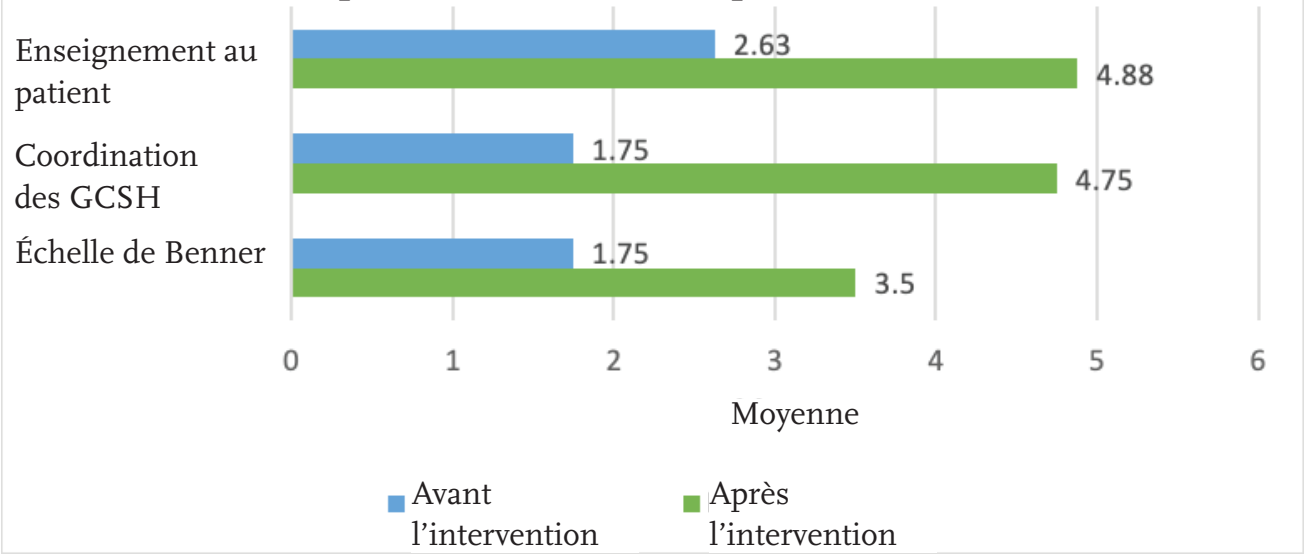


Figure 7. Changement du stade de compétence du modèle de Benner avant et après le programme d'orientation

\section{Stade du modèle de Benner}

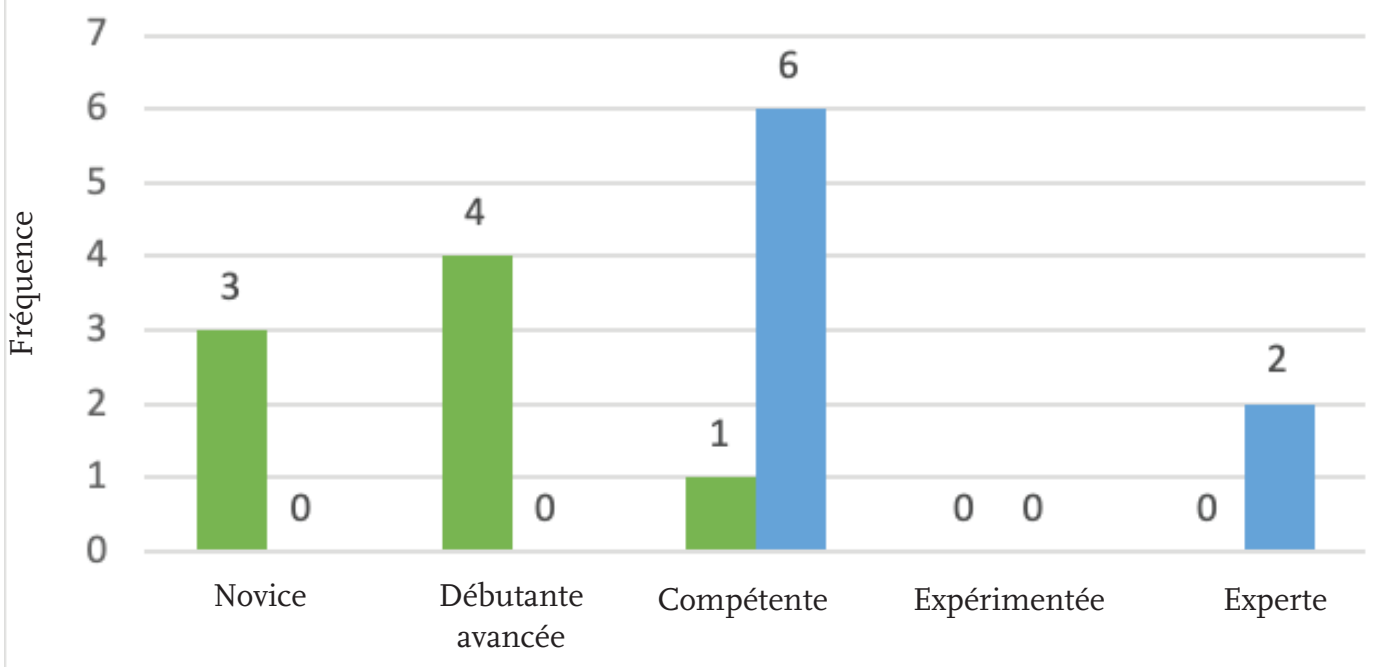

Avant l'intervention $\square$ Après l'intervention

pas été intégré à l'étude. Létude serait plus complète si nous connaissions les effets du programme d'orientation sur les résultats mesurables de la GCSH. Toutefois, il s'agit d'une question complexe et les facteurs d'influence sont multiples; il serait difficile d'isoler l'effet causé directement par l'orientation du personnel de coordination. Il serait possible d'étayer l'étude en évaluant de nouveau les participantes 4 à 6 mois après l'orientation pour vérifier la rétention des connaissances et des compétences. L'ajout d'une composante qualitative à l'évaluation permettrait de mieux comprendre comment les participantes perçoivent les bénéfices du programme d'orientation au fil du temps.

D'autres conditions, comme des facteurs en lien avec le préceptorat ou à d'autres activités de formation continue que les apprenantes suivent de leur propre initiative pendant la période d'orientation, pourraient influencer l'évolution des connaissances et comportements avant et après l'orientation. Pour clarifier ce point, il faudrait que d'autres études se penchent sur l'utilisation d'un parcours d'apprentissage dans l'orientation des coordonnatrices de GCSH.

Dans la présente étude, on a recueilli les données par auto-évaluation. Cette méthode peut toutefois être biaisée, car les répondants ont tendance à surévaluer leurs réponses (Leggett et al., 2016). Pour atténuer cet effet, l'étude utilisait les mêmes questionnaires avant et après l'intervention et analysait l'ampleur du changement au fil du temps. De plus, pour éviter une possible pression de la part du groupe, les questionnaires étaient anonymes.

L'auto-évaluation des connaissances portait sur les éléments suivants : 1) la procédure de greffe; 2) son rôle dans la trajectoire des soins du patient; 3) les étapes de soins au patient pendant le processus de greffe; et 4) l'organigramme de coordination. Chaque élément était évalué par une seule affirmation notée sur une échelle de Likert. Peut-être que l'utilisation de multiples affirmations pour chaque domaine de connaissances améliorerait la sensibilité de la mesure à rendre compte de l'évolution. Par exemple, plutôt qu'une seule affirmation sur la procédure de GCSH, certaines questions plus pointues pourraient aborder la préparation, l'injection des cellules, les complications immédiates et les complications tardives.

Enfin, huit infirmières d'un seul centre de GCSH ont participé à la présente étude; il s'agit d'un échantillon relativement petit, mais typique d'un domaine de pratique hautement spécialisé comme la GCSH. Les résultats de l'étude devront être confirmés dans d'autres centres de greffe.

\section{CONCLUSION}

La présente étude avait pour objectif d'évaluer l'efficacité d'un parcours d'apprentissage pour orienter les nouvelles coordonnatrices de greffes du CSSH. Selon les résultats obtenus, il s'agit d'une méthode efficace pour accroître les connaissances et renforcer les comportements des infirmières en orientation pour le rôle de coordonnatrice. À la suite de la période d'orientation, organisée selon le parcours d'apprentissage, les nouvelles coordonnatrices ont perçu une amélioration de leur niveau d'aptitude selon le modèle des compétences infirmières de Benner (1984). Les documents d'orientation connexes, la trousse d'étude et l'outil d'évaluation des compétences ont tous eu une influence positive sur les résultats.

De plus en plus de patients ont besoin d'une greffe de cellules souches hématopoiétiques. Par conséquent, dans de nombreux programmes de greffes, la coordination est un rôle en pleine expansion. Auparavant, ce domaine de spécialité ne 
disposait pas d'un programme d'orientation formel. S'inspirant de méthodes éprouvées dans d'autres domaines pour orienter les médecins et les infirmières, la présente étude, réalisée dans un centre de GCSH, a permis de constater l'utilité du parcours d'apprentissage en coordination des greffes, ainsi que de la trousse d'étude et de l'outil d'évaluation des compétences qui l'accompagnaient, pour augmenter considérablement le niveau de connaissances et de compétences du personnel. Des études ultérieures dans d'autres centres de GCSH devront être

\section{RÉFÉRENCES}

Benner, P. (1984). From novice to expert: Excellence and power in clinical nursing practice. Addison-Wesley

Boyd, V. A., Whitehead, C. R., Thille, P., Ginsburg, S., Brydges, R., \& Kuper, A. (2018). Competency-based medical education: The discourse of infallibility. Medical Education, (1), 45. https://doi. org/10.1111/medu.13467

Bumgarner, S., \& Biggerstaff, G. (2000). A patient-centered approach to nurse orientation. Journal for Nurses in Staff Development, 16(6), 249-256.

Canadian Association of Nurses in Oncology. (2002). Practice standards and competencies. http://c.ymcdn.com/sites/cano. malachite-mgmt.com/resource/resmgr/standards/CONEP_ Standards2006September.pdf

CCO Complex Malignant Hematology Hematopoietic Cell Therapy Consultation Group (2017). Complex malignant hematology services in Ontario June 2017 - Year in review. https://www.cancercare. on.ca/common/pages/UserFile.aspx?fileId=381199

Ezzone, S. (2013). Hematopoietic stem cell transplantation: A manual for nursing practice (2nd ed.). Oncology Nursing Society. ISBN: 9781935864196.

Fastre, G. M. J., van der, Klink, M. R., Amsing-Smit, P., \& van Merriënboer, J. J. G. (2014). Assessment criteria for competencybased education: A study in nursing education. Instructional Science, 42(6), 971-94.

Greet M. J., F., Marcel R. van der, K., Pauline, A., \& Jeroen, J. G. van, M. (2014). Assessment criteria for competency-based education: a study in nursing education. Instructional Science, (6), 971.

Halter, C.P. (2017). The PSPP Guide: An introduction to statistical analysis. Creative Minds Press Group.

Harpe, S.E. (2015). How to analyze Likert and other rating scale data. Currents in Pharmacy Teaching Q Learning, (7), 836-850.

Hertzog, M. A. (2008). Considerations in determining sample size for pilot studies. Research in Nursing \& Health, 31(2), 180-191. https:// doi.org/10.1002/nur.20247

Israel, D. G. (2003). Determining sample size. University of Florida IFAS Extension. https://www.tarleton.edu/academicassessment/ documents/Samplesize.pdf menées pour corroborer l'efficacité du parcours d'apprentissage intégré pour l'orientation dans ce domaine de spécialité et vérifier si les résultats obtenus sont applicables à plus grande échelle.

\section{REMERCIEMENTS}

Nous aimerions remercier Tammy DeGelder, inf. aut. (catégorie spécialisée), d'avoir aidé à recueillir le consentement des participants au projet de recherche.

Johnston, P., \& Ferraro, C. (2001). Application of critical pathways in the maternity nursing orientation process. Journal or Nurses in Staff Development, 17(2), 61-66.

Kirkpatrick, D. L. (2006). Evaluating training programs: The four levels ( $3^{\text {rd }}$ edition). Berrett-Koehler.

Leggett, L. E., Khadaroo, R. G., Holroyd-Leduc, J., Lorenzetti, D. L., Hanson, H., Wagg, A., Padwal, R., \& Clement, F. (2016). Measuring resource utilization: A systematic review of validated self-reported questionnaires. Medicine, 95(10), e2759. https://doi.org/10.1097/ MD.0000000000002759

Majhail, N. S., Mau, L. W., Chitphakdithai, P., Payton, T., Eckrich, M., Joffe, S., Lee, S. J., LeMaistre, C. F., LeRademacher, J., Loberiza, F., Logan, B., Parsons, S. K., Repaczki-Jones, R., Robinett, P., Rizzo, J. D., Murphy, E., \& Denzen, E. M. (2015). Clinical research: Supportive care: National survey of hematopoietic cell transplantation center personnel, infrastructure, and models of care delivery. Biology of Blood and Marrow Transplantation, 2113081314. https://doi.org/10.1016/j.bbmt.2015.03.020

Miglore, G., Boeding, M., Barut, J., \& Harris, L. (2017). Nurses initiating change: Engaging nurses in development of unit orientation pathways and processes at an inpatient psychiatric hospital. Journal of The American Psychiatric Nurses Association [serial online], 23(4), 297-298. Science Citation Index.

Meek, G., Ozgur, C., \& Dunning, K. (2007). Comparison of the t vs. Wilcoxon signed-rank test for Likert scale data and small samples. Journal of Modern Applied Statistical Methods, 6(1), 91-106.

Schumacher, G., \& Risco, K. (2017). Competency-based nurse practitioner education: An overview for the preceptor. Journal for Nurse Practitioners, (9), 596. https://doi.org/10.1016/j. nurpra.2017.07.020

Sullivan, G. M., \& Artino, A. R., Jr. (2013). Analyzing and interpreting data from Likert-type scales. Journal of Graduate Medical Education, 5(4), 541-542. https://doi.org/10.4300/JGME-5-4-18 